\title{
Implication of leucyl-tRNA synthetase 1 (LARS1) over-expression in growth and migration of lung cancer cells detected by siRNA targeted knock-down analysis
}

\author{
Seung-Hun Shin ${ }^{1,2 *}$, Ho-Shik Kim ${ }^{3 *}$, \\ Seung-Hyun Jung ${ }^{1,2}$, Hai-Dong $\mathrm{Xu}^{1,2}$, \\ Yong-Bok Jeong ${ }^{1,2}$ and Yeun-Jun Chung ${ }^{1,2,4}$

\section{'Department of Microbiology} \\ ${ }^{2}$ Integrated Research Center for Genome Polymorphism \\ ${ }^{3}$ Department of Biochemistry \\ College of Medicine, The Catholic University of Korea \\ Seoul 137-701, Korea \\ ${ }^{4}$ Corresponding author: Tel, 82-2-590-1214; Fax, 82-2-596-8969; \\ E-mail, yejun@ catholic.ac.kr \\ *These authors contributed equally to this work.
}

Accepted 24 January 2008

Abbreviations: ACP, annealing control primer; ARS, aminoacyltRNA synthetase; DEG, differentially expressed gene; LARS1, leucyl-tRNA synthetase 1; siRNA, small interfering RNA

\begin{abstract}
Molecular mechanism of lung carcinogenesis and its aggressive nature is still largely elusive. To uncover the biomarkers related with tumorigenesis and behavior of lung cancer, we screened novel differentially expressed genes (DEG) in A549 lung cancer cell line by comparison with CCD-25Lu, normal pulmonary epithelial cell line, using annealing control primer(ACP)based GeneFishing system. Of the DEGs, over-expression of leucyl-tRNA synthetase 1 (LARS1) was prominent and this up-regulation was confirmed by immunoblotting and real-time quantitative RT-PCR analysis. In addition to $\mathbf{A 5 4 9}$ cell line, primary lung cancer tissues also expressed higher level of LARS1 mRNA than their normal counter tissues. To explore the oncogenic potential of LARS1 over-expression in lung cancer, we knocked-down LARS1 by treating siRNA and observed the tumor behavior. LARS1 knock-down cells showed reduced ability to migrate through transwell membrane and to form colonies in both soft agar and culture plate. Taken together, these findings suggest that LARS1 may play roles in migration and growth of lung cancer cells, which suggest its potential implication in lung tumorigenesis.
\end{abstract}

Keywords: amino acyl-tRNA synthetases; cell movement; cell proliferation; leucine-tRNA ligase; lung neoplasms; oncogenes; RNA, small interfering

\section{Introduction}

Lung cancer is the most common and the leading cause of cancer death in the world (Boyle and Ferlay, 2005). Current staging system for lung cancer is inadequate for predicting the outcome of treatment and lack of efficient early screening tool for lung cancer is one of obstacles to improve the treatment and prevention of lung cancer.

The search for biologic markers of lung cancer has been developed to apply them for clinical applications during last decades (Nguyen and Schrump, 2006). In addition to imaging technology, genetic biomarkers are useful in diagnosis and therapeutic interventions for lung cancers. Large numbers of altered expression-based biomarkers have been reported in lung cancer (Greenberg and Lee, 2007; Kwon et al., 2007). Among them, carcinoembryonic antigen (CEA) (Okada et al., 2004), CYFRA 21-1 (Kulpa et al., 2002), neuron-specific enolase (NSE) (Ferrigino et al., 2003), squamous cell carcinoma (SCC) antigen (Schneider, 2006), tumor M2-pyruvate kinase (Tumor M2-PK) (Schneider, 2006) and c-reactive protein (CRP) (Siemes, et al., 2006) have been well studied. Genomic aberrations are another frequently observed event in lung cancer (Testa et al., 1997; Balsara and Testa, 2002). Potentially novel cancer related genes have been identified in the recurrently altered genomic regions in lung cancers by using whole-genome copy number analysis methods such as array-comparative genomic hybridization (array-CGH) (Kim et al., 2005). However, early detection and prediction of treatment outcome is still largely elusive in lung cancer.

Recent development of high throughput gene expression profiling technologies such as wholegenome expression microarray and random primed RT-PCR have facilitated mining novel differentially expressed genes (DEGs) in many cancers. Especially the differential display method based on annealing control primers (ACP) overcomes shortcomings of conventional differential display RT- 
PCR (DDRT) or suppression subtractive hybridization (Hwang et al., 2003; Kim et al., 2004; MüllerHagen et al., 2004).

To explore the tumorigenesis of lung cancer and identify efficient biomarkers, we screened the DEGs in A549 lung cancer cell line by using APC based DEG detection system. Among the up or down regulated candidate transcripts we validated the oncogenic potential of LARS1 gene, one of the prominently up-regulated transcript in A549, by siRNA knockdown and various transformation assays. We also observed the expression profile of $L A R S 1$ in primary lung cancer samples.

\section{Materials and Methods}

\section{Cell culture}

A549 lung cancer cell line (CCL-185) and CCD25Lu normal pulmonary epithelial cell line (CCL215) purchased from American Type Culture Collection (ATCC, Manassas, VA) were maintained in RPMI1640 medium containing 10\% FBS (Hyclone, Logan, UT) and $10 \mathrm{U} / \mathrm{ml}$ of penicillin-streptomycin (Sigma, St. Louis, MO) under $5 \% \mathrm{CO}_{2}$.

\section{Screening of differentially expressed genes (DEGs)}

For screening the DEGs in A549 cell, we used ACP-based GeneFishing PCR kit (Seegene, Seoul, Korea) according to manufacturer's instruction. In brief, first-strand cDNAs were synthesized with total RNA extracted from CCD-25Lu and A549 cell lines using Moloney murine leukemia virus reverse transcriptase (Promega, Madison, WI) and dTACP1 (5'-CTGTGAATGCTGCGACTACGATIIIIIT(18)$\left.3^{\prime}\right)$ primer. Second-strand CDNA was synthesized using primers of dT-ACP2 and arbitrary ACP with one cycle reaction as follows; $94^{\circ} \mathrm{C}$ for $1 \mathrm{~min}, 50^{\circ} \mathrm{C}$ for $3 \mathrm{~min}$, and $72^{\circ} \mathrm{C}$ for $1 \mathrm{~min}$, sequentially. Following second-strand cDNA synthesis, the secondstage PCR amplification was carried out as follows; 40 cycles of $94^{\circ} \mathrm{C}$ for $40 \mathrm{~s}, 65^{\circ} \mathrm{C}$ for $40 \mathrm{~s}$ and $72^{\circ} \mathrm{C}$ for $40 \mathrm{~s}$, followed by 5 -min final extension at $72^{\circ} \mathrm{C}$. The amplified PCR products were separated in $2 \%$ agarose gel containing ethidium bromide. The amplified cDNA fragments with differential band intensities were re-amplified and extracted from the gel by using the GENCLEAN II Kit (Q-BIO gene, Carlsbad, $C A$ ), and directly sequenced with $A B I$ PRISM 310 Genetic Analyzer (Applied Biosystems, Foster City, CA).

\section{Real-time quantitative RT-PCR (qRT-PCR)}

The extracted total RNA was reversely transcribed to cDNA using oligo-(dT) primer and SuperScript II reverse transcriptase (Invitrogen, Carlsbad, CA). The $\mathrm{qRT}$-PCR was performed with mixture containing cDNA, $1 \times$ SYBR Green Tbr polymerase mixture (FINNZYMES, Finland), $\operatorname{ROX}(0.5 \times)$ and primers using Mx3000P QPCR (Stratagene, La Jolla, CA). GAPDH was used as an internal control in each procedure. The thermal cycling was one cycle at $95^{\circ} \mathrm{C}$ for $10 \mathrm{~min}$, followed by 40 cycles of $95^{\circ} \mathrm{C}$ at $10 \mathrm{~s}, 60^{\circ} \mathrm{C}$ for $30 \mathrm{~s}$ and $72^{\circ} \mathrm{C}$ for $30 \mathrm{~s}$. After amplifying $\mathrm{PCR}$ reaction, melting curve analysis was performed from $55^{\circ} \mathrm{C}$ to $95^{\circ} \mathrm{C}\left(\Delta 0.5^{\circ} \mathrm{C} / \mathrm{s}\right)$. $L A R S 1$ specific primers were as follows; forward primer 5'-ATGGCGGAAAGAAAAGGAACAG-3' and reverse primer 5'-CAGGCCAAAGGGAAACAGACAAC-3'. GAPDH specific primers were as follows; forward primer 5'-GCGGGGCTCTCCAGAACATCAT-3' and reverse primer 5'-CCAGCCCCAGCGTCAAAGGTG-3'. Relative quantification was performed by the $\Delta \Delta \mathrm{Ct}$ method (Livak and Schmittgen, 2001). The signal intensity ratio (LARS1/ $G A P D H)$ of the tumor sample was compared with that of the normal lung tissue. All qRT-PCR experiments were repeated twice and represented as mean of ratio (tumor/normal) $\pm S D$. In case of qRTPCR for primary lung cancers, we performed single experiment due to limitation of samples.

\section{Knock-down of LARS1 expression}

Stealth small interfering RNA (siRNA) against LARS1 was synthesized as follows; 5'-AAAUGAAGGCGUCCAUUCAUAUAUG-3'. Scrambled RNA for negative control was purchased from Invitrogen. For knocking-down of LARS1 expression, A549 cells were plated at a concentration of 400,000 cells/well in 6-well plates and incubated overnight. The cells were transfected with $50 \mathrm{nM}$ siRNA using Lipofectamine 2000 (Invitrogen). After $10 \mathrm{~h}$ of initial incubation, we replaced the medium with fresh medium and incubated for $14 \mathrm{~h}$.

\section{Western blot analysis for LARS1}

Cellular proteins (30 $\mu \mathrm{g}$ per lane) were electrophoresed in 10\% SDS-polyacrylamide gel. Separated proteins were transferred to a PVDF membrane, which was blocked with $5 \%$ non-fat dried milk in TBST (20 mM Tris- $\mathrm{HCl}, 150 \mathrm{mM} \mathrm{NaCl}$, and $0.1 \%$ Tween 20, pH 7.5), and then incubated overnight with anti-LARS1 antibody (Abcam, Cambridge, UK) and anti $\beta$-actin antibody (Sigma, St. Louis, MI). After washing with TBST, the PVDF membrane was incubated with diluted HRP-conjugated anti-rabbit $\lg$ for $1 \mathrm{~h}$ at room temperature. The blots were then detected using enhanced 
chemiluminescence (ECL) system (AmershamPharmacia Biotech, Braunschweig, Germany).

\section{Colony formation assay}

Colony formation assay was performed to explore the effect of LARS1 knock-down in A549 as described previously (Crnković-Mertens et al., 2006). Twenty hours after transfection with siRNA, A549 cells were seeded in 6-well plates. After 6-day growing, cells were washed with PBS and stained with $0.5 \%$ crystal violet in $20 \%$ methanol. Cell colonies larger than $1 \mathrm{~mm}$ in diameter were counted under a microscopic field at $40 \times$ magnification.

\section{Soft agar assay}

To explore the effect of LARS1 on anchorageindependent growth of A549 cells, soft agar assay was performed as described previously (Zhang et al., 2006). Twenty hours after transfection with siRNA, 1,000 cells in $0.35 \%$ low-melting agarose with RPMI1640 were plated in each well on the top of existing $0.6 \%$ bottom low-melting agarose in six-well culture plate. After incubating for $2 \mathrm{wk}$ at $37^{\circ} \mathrm{C}$ in a $5 \% \mathrm{CO}_{2}$, colonies were counted by the same method for colony formation assay.

\section{Migration assay}

Migration assay was performed as described previously (Lee et al., 2007) using 24-well format transwell chambers (Becton Dickinson Labware, Franklin Dickinson, NJ), $8 \mu \mathrm{m}$-pore size, with slight modifications. In brief, A549 cells suspended in $400 \mu \mathrm{l}$ serum-free RPMI1640 were seeded onto upper part of each chamber, whereas the lower compartments were filled with $500 \mu \mathrm{l}$ of RPMI1640 containing $10 \%$ FBS. After incubation for $24 \mathrm{~h}$ at $37^{\circ} \mathrm{C}$, cells were stained with $0.5 \%$ crystal violet in $20 \%$ methanol. Non-migrated cells on the upper surface of the filter were wiped out with cotton swab. Migrated cells were counted in 10 microscopic fields with $5 \times$ objective lens per well.

\section{RESULT}

\section{DEGs in lung cancer cell lines}

To identify DEGs at lung cancer cell lines, we employed ACP-based GeneFishing PCR and compared the band intensities between A549 and CCD-25Lu cell lines. By densitometry analysis of amplified cDNA fragments, we selected 8 fragments among the bands showing different intensities between two cell lines. The eight amplicon bands were eluted from agarose gel, re-amplified and sequenced. The sequences were used for BLAST search to identify their gene annotations. According to the BLAST analysis, six were known genes and the other two were unknown genes (Table 1). Among those 6 genes, we selected LARS1 for further functional evaluation of its oncogenic potential, because the expression level was highly different between A549 and CCD25-Lu and several members of aminoacyl-tRNA synthetase (ARS) family were reported to be involved in diverse biological processes. Figure $1 \mathrm{~A}$ illustrates the GeneFishing result for LARS1.

\section{Over-expression of $L A R S 1$ in $A 549$ cells and primary lung cancer tissues}

To confirm the relatively higher expression of LARS1 in A549 cells detected by GeneFishing, we

Table 1. Summary of genes differentially expressed between CCD-25L and A549 cell lines.

\begin{tabular}{|c|c|c|c|c|}
\hline ID & Gene annotation $^{a}$ & Accession No. & Expect value $^{b}$ & DEG profile ${ }^{c}$ \\
\hline DEG1 & Transketolase & BC002433 & 0.0 & High \\
\hline DEG2 & Leucyl-tRNA synthetase (LARS1), & NM020117 & $7 e-134$ & High \\
\hline DEG3 & Lysyl oxidase-like 1 (LOXL1) & BC068542 & 0.0 & Low \\
\hline DEG4 & Proteasome (prosome, macropain) 26S subunit & BC010396 & 0.0 & High \\
\hline DEG5 & Myofibrillogenesis regulator 1 & BC002937 & $2 \mathrm{e}-162$ & High \\
\hline DEG6 & v-myc myelocytomatosis viral oncogene homolog & BC058901 & $1 e-147$ & Low \\
\hline DEG7 & No significant & & $2 \mathrm{e}-117$ & Low \\
\hline DEG8 & No significant & & $5 e-141$ & Low \\
\hline
\end{tabular}

a. The sequencing result of the candidate cDNA amplicon bands were used for BLAST search to identify their annotations.

b. E-value; a parameter that describes the number of hits one can expect to see by chance when searching a database of a particular size.

c. Relatively higher or lower-expression in A549 compared with CCD-25Lu normal pulmonary epithelial cell. 
A

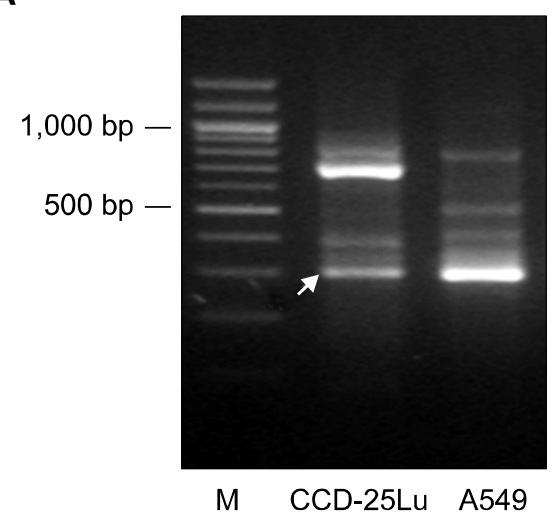

B

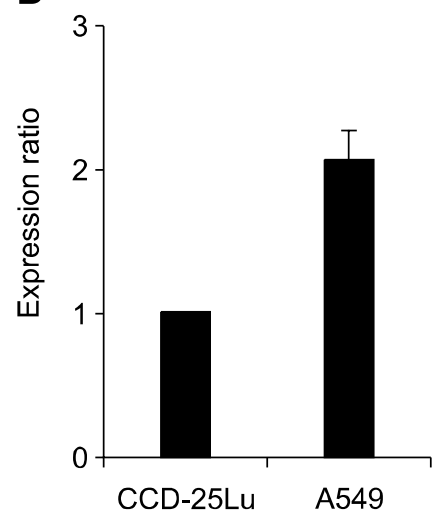

C

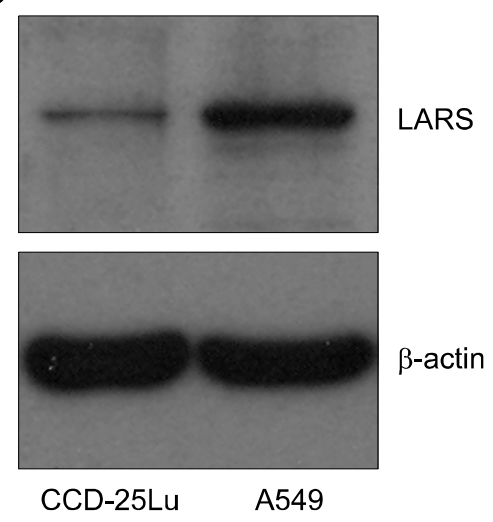

D

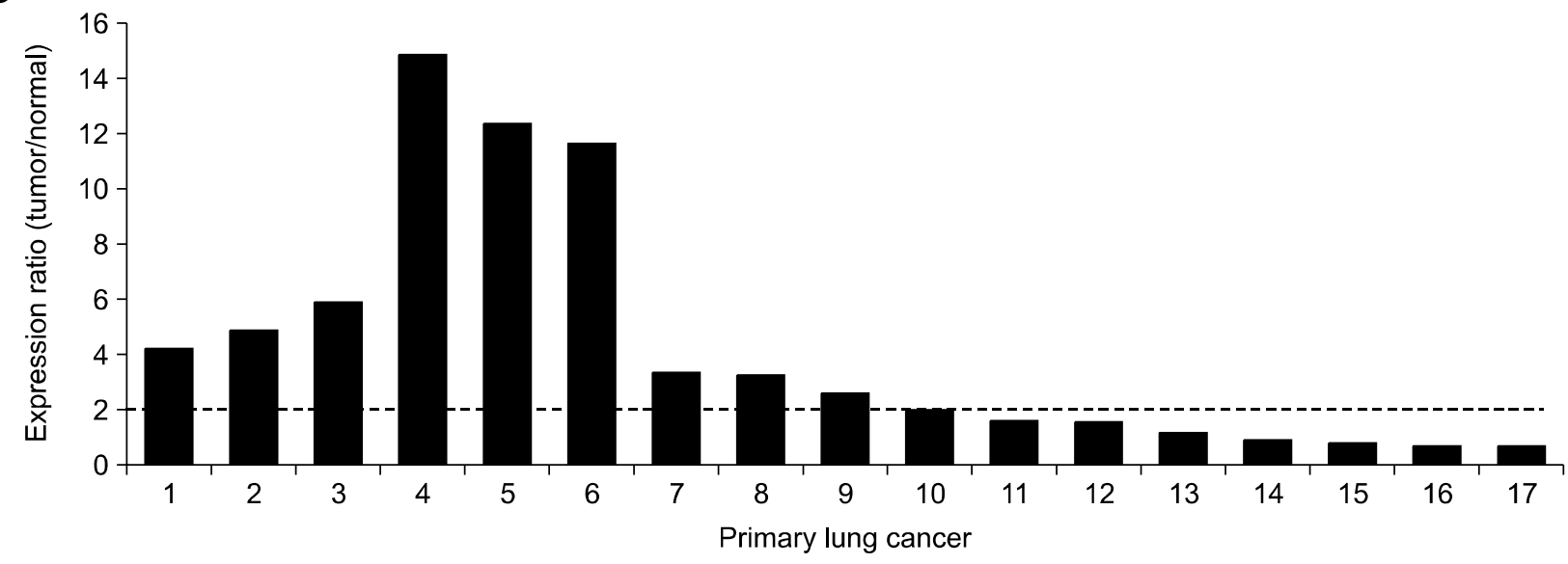

Figure 1. Over-expression of LARS1 gene in lung cancer cells. (A) Total RNA of CCD-25Lu and A549 cells were used for ACP-based GeneFishing analysis as described in Materials and Methods. The amplicon bands showing different intensities between two cell lines were re-amplified and sequenced for gene annotation. Arrow indicates a DEG band turned out to be LARS1. (B) Expression level of LARS1 in each cell was determined by qRT-PCR as described in Materials and Methods. As an internal control, GAPDH mRNA was used. (C) Cell lysates of CCD-25Lu and A549 cells were used for immunoblot analysis with anti-LARS1 antibody. $\beta$-actin was probed for equal protein loading. (D) Expression level of LARS1 in 17 primary lung cancer samples was determined by qRT-PCR as described in Materials and Methods. Ten of the 17 primary cancers showed over two fold up-regulation of LARS1 (criteria of over-expression in this study) compared to normal control lung tissues.

screened LARS1 expression using qRT-PCR and immunoblot analysis in the same cell line. qRTPCR experiments were repeated twice and represented as mean of ratio (tumor/normal) \pm SD. As shown in Figure 1B, RNA expression level of LARS1 in A549 cells was more than two-fold higher $(2.06 \pm 0.19)$ than that in CCD-25Lu. LARS1 protein level was also higher in A549 than in CCD$25 \mathrm{Lu}$ (Figure 1C). These findings suggest that LARS1 is generally over-expressed in lung cancers. To examine this possibility, we checked the mRNA expression levels of LARS1 in 17 primary lung cancer tissues by qRT-PCR (Figure 1D). In 10 out of 17 lung cancer tissues, the expression of LARS1 mRNA was increased more than two-fold compared to their normal counter tissues, while there was no primary lung cancers under-ex- pressed below two-fold. This data indicates that LARS1 is commonly over-expressed in lung cancers.

\section{The effect of siRNA against LARS1 on tumor cell growing}

SiRNA against LARS1 (siLARS) repressed the expression of LARS1 at both mRNA (tumor/normal ratio $=0.33 \pm 0.21$ ) and protein levels compared to those in cells treated with control siRNA (siCon) (Figure 2A and $\mathrm{B}$ ). Then, we checked the effect of LARS1 down-regulation on A549 cell growth. In colony formation assay, colony numbers of siLARStreated A549 cells were reduced to $64 \%( \pm 14 \%)$ compared to siCon-treated cells (Figure $3 A$ ). In soft agar assay, colony numbers of siLARS-treated 
A

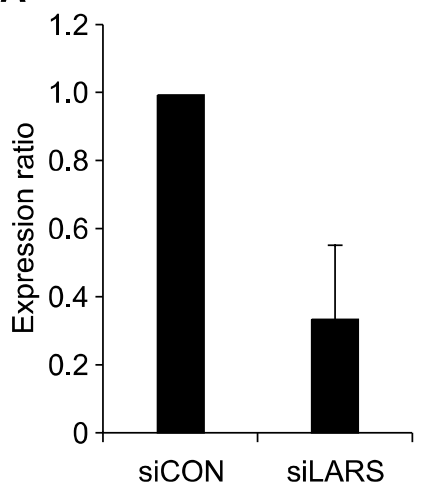

B

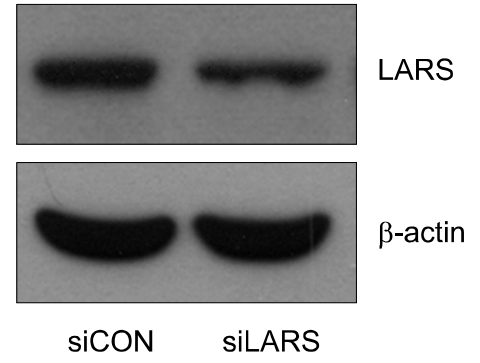

Figure 2. Down-regulation of LARS1 expression in A549 cells by treating siRNA against LARS1 (siLARS). (A) A549 cells were transfected with $50 \mathrm{nM}$ of siLARS or siCon and incubated for 14 hours. Then LARS1 expression was compared between siLARS and siCon treated cells by qRT-PCR as described in Materials and Methods. As an internal control, GAPDH mRNA was used. (B) LARS1 protein expression was compared between the same cells by immunoblot analysis. $\beta$-actin was probed for equal protein loading.
A549 cells were reduced to below half (43 \pm $9.5 \%$ ) of those of siCon-treated cells and the colony size was also generally smaller in siLARStreated cells than in siCon-treated cells (Figure 3B).

\section{The effect of siLARS on tumor cell migration}

We also examined the effect of siLARS on the cancer cell migration using transwell assay. A549 cells transfected with siLARS showed clearly decreased migratory activity (62 $\pm 21.2 \%$ ) compared to cells treated with siCon (Figure $3 \mathrm{C}$ ), suggesting the contribution of LARS1 over-expression on cancer cell migration.

\section{Discussion}

In this study, we suggest that LARS1, a member of ARS family, involves in lung tumorigenesis and tumor cell behavior by influencing tumor cell growth and migration. Consistent up-regulation of LARS1 in both DEG and qRT-PCR shows the reliability of ACP-based GeneFishing analysis, which we employed in this study. We observed the higher expression of LARS1 in lung cancer cell line as well as primary lung cancer tissues. For example, in addition to A549 cell line, 59\% (10/17) of primary lung cancers showed more than two-fold up-regulation of LARS1, which suggests its oncogenic potential in lung cancer. We hypothesized that if over-expression of LARS1 contributes tumor behavior of lung cancer such as tumor growth and migration, knocking-down the over-expressed LARS1 could suppress those phenotypes. As we expected, we clearly observed the inhibitory effect on lung cancer cell growth and migration by siLARS treatment, which confirms its oncogenic role.

The ARS family members are housekeeping enzymes to catalyze the aminoacylation of tRNA during polypeptide synthesis. Recently, ARS was reported to have many other non-catalytic activities such as apoptosis, angiogenesis, RNA splicing and inflammation in addition to its housekeeping function for protein synthesis (reviewed in Lee et al., 2004). For example, human QARS interacts with apoptosis signal-regulating kinase 1 (ASK1) and blocks its kinase activity leading to inhibition of cell death (Ko et al., 2001). N-terminal fragment of human YARS (called mini-YARS) generated from full length YARS by leukocyte elastase was reported to bind strongly to the CXC-chemokine receptor CXCR1, serving as a chemo-attractant for polymorphonuclear leukocytes and to induce angiogenesis (Wakasugi et al., 2002). By contrast, $\mathrm{N}$-terminally truncated form of human WARS reported to inhibit angiogenesis in response to IFN- $\gamma$ (Otani et al., 2002; Kise et al., 2004). In yeast, LARS2 (mitochondrial LARS) was also reported to have a non-canonical activity with splicing of group I introns (Labouesse, 1990; Rho et al., 2002). However, there has been no report about noncanonical activity of human LARS, irrespective of its localization; cytoplasm or mitochondria. So, this report, to our knowledge, is the first evidence showing the non-canonical activity of human LARS.

To figure out the molecular mechanisms involved in the inhibitory effect of siLARS on tumor cell growth and migration, we observed the expression profile of the proteins involved in cell cycle and cell motility such as p53, c-Myc, matrix metalloproteinase (MMP)-1 and MMP-2 in LARS1 knock-down cells. But, there was no clear difference of those proteins by LARS1 knock-down (data not shown). Signal transducers or modulators required for cell survival and migration including signal transducer and activator of transcription (STAT) family members and AKT kinase were not perturbed either (data not shown). Further comprehensive downstream analysis such as global expression profiling or proteomic screening will be 
A

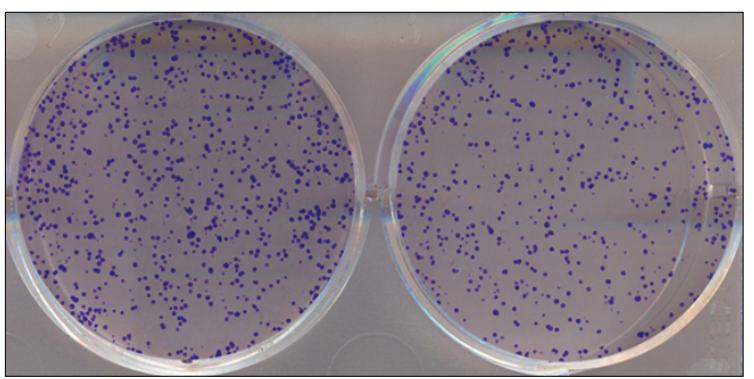

siCON

siLARS

B

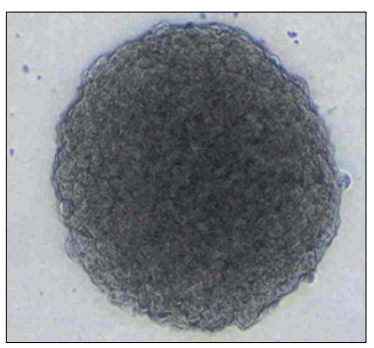

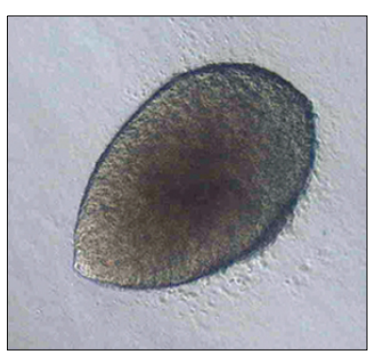

siCON
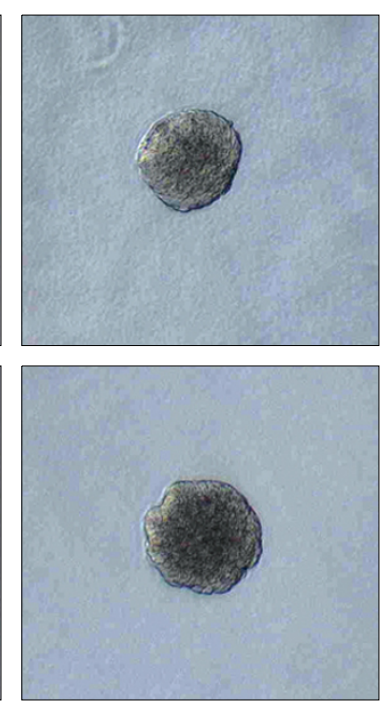

siLARS

C

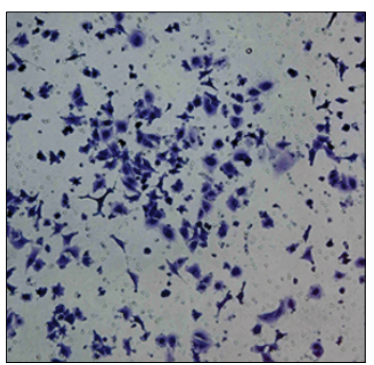

siCON

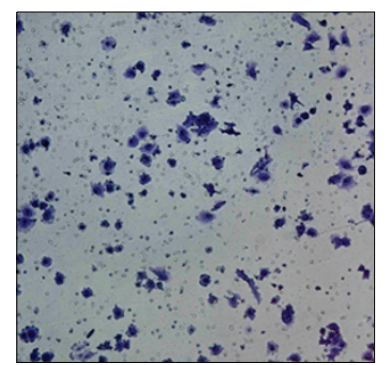

siLARS
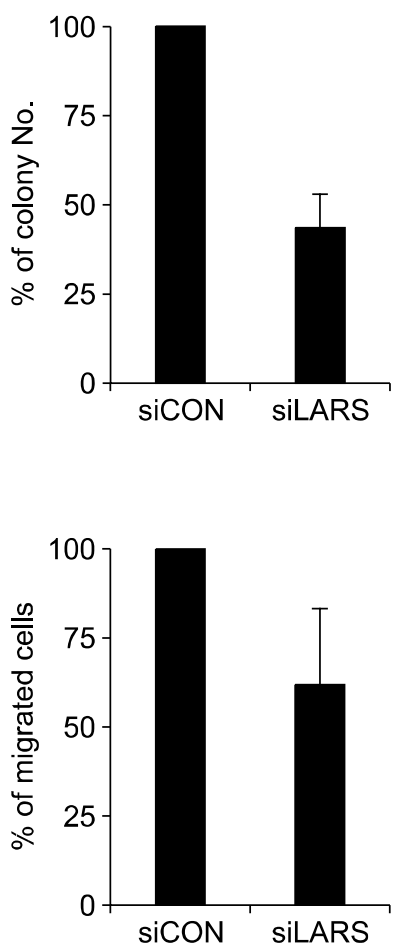

Figure 3. Effect of siLARS on colony formation and migration of A549 cells. (A) Colony formation assay. A549 cells were transfected with siCon and siLARS, respectively, and incubated in 6-well plates for 6 days. Colonies larger than $1 \mathrm{~mm}$ in diameter were counted. Bar chart in the right box represents the percentage of colony numbers in siLARS-treated cells to those in siCon-treated cells. Data represents mean of percentages $\pm S D$ of three independent experiments. (B) Soft agar assay. Same cells were incubated in low-melting agarose as described in Materials and Methods. Two weeks later, colonies were photographed and numbers of colonies were counted. (C) Transwell assay. Same cells were incubated in upper chamber of 24-well transwell chambers for migration assay. After $24 \mathrm{~h}$, migrated cells were counted as described in Materials and Methods. The migrated cells were photographed and percentages of migrated siLARStreated cells to migrated siCon-treated cells were calculated. Data represents mean of percentages \pm SD of three dependent experiments. required to explore the molecular mechanisms of siLARS mediated anti-tumorigenic activity.

The rate of protein synthesis in cancer cells is generally higher than that in normal cells because cancer cells proliferate more extensively and their metabolism is more dynamic than normal cells. So, it can be assumed that ARS would be elevated in cancer cells including lung cancer. Given that the assumption, the attenuation of lung cancer cell growth and migration by LARS1 knock-down might be attributable to general, non-specific inhibition of protein synthesis. However, none of ARS family members except LARS1 appeared to be elevated in lung cancer cells by DEG analysis and moreover, expression of several proteins involved in cancer cell growth and migration described above 
were not changed by knock-down of LARS1, which suggest that the effect of LARS1 knock-down might not due to general inhibition of protein synthesis but more likely LARS1-specific mechanism.

Our study has several limitations. For example, we have not observed the global expression or proteomics profile in the LARS1 knock-down cells, which may provide clues to understand the signal transduction pathway leading to LARS1 over-expression and the molecular mechanism of influencing lung cancer growth and migration via overexpression of LARS1. Secondly, although we observed the LARS1 expression in 17 primary lung cancers, more large scale screening will be required to confirm its role in primary cancers.

In conclusion, we identified the over-expression of $L A R S 1$, a member of ARS family, in lung cancer cell lines and primary lung cancers. We suggested its potential implication in lung tumorigenesis and tumor behavior by influencing tumor cell growth and migration. To our knowledge, this is the first report showing frequent over-expression of LARS1 in both lung cancer cell line and primary tumors, and suggesting its oncogenic potential. Taken together, our results and the strategy will be helpful to elucidate pathogenesis of lung cancer and to develop biomarkers.

\section{Acknowledgements}

This study was supported by a grant from the Korea Health 21 R\&D Project, Ministry of Health and Welfare, Republic of Korea (01-PJ3-PG6-01GN07-0004).

\section{References}

Balsara BR, Testa JR. Chromosomal imbalances in human lung cancer. Oncogene 2002;21:6877-83

Boyle P, Ferlay J. Cancer incidence and mortality in Europe. Ann Oncol 2005;16:481-8

Crnković-Mertens I, Muley T, Meister M, Hartenstein B, Semzow J, Butz K, Hoppe-Seyler F. The anti-apoptotic livin gene is an important determinant for the apoptotic resistance of non-small cell lung cancer cells. Lung Cancer 2006; $54: 135-42$

Ferrigino D, Bucheri G, Giordano C. Neuron-specific enolase is an effective tumor marker in nonsmall cell lung cancer (NSCLC). Lung Cancer 2003;41:311-20

Greenberg AK, Lee MS. Biomarkers for lung cancer: clinical uses. Curr Opin Pulm Med 2007;13:249-55

Hwang IT, Kim YJ, Kim SH, Kwak CI, Gu YY, Chun JY. Annealing control primer system for improving specificity of PCR amplification. BioTechniques 2003;35:1180-4

Kim YJ, Kwak Cl, Gu YY, Hwang IT, Chun JY. Annealing control primer system for identification of differentially expressed genes on agarose gels. BioTechniques 2004;36: 424-6

Kim TM, Yim SH, Lee JS, Kwon MS, Ryu JW, Kang HM, Fiegler H, Carter NP, Chung YJ. Genome-wide Screening of Genomic Alterations and Their Clinicopathological Implications in Non Small Cell Lung Cancers. Clinical Cancer Research 2005;11:8235-42

Kise Y, Lee SW, Park SG, Fukai S, Sengoku T, Ishii R, Yokoyama S, Kim S, Nureki O. A short peptide insertion crucial for angiostatic activity of human tryptophanyl-tRNA synthetase. Nat Struct Mol Biol 2004;11:149-56

Ko YG, Kim EY, Kim T, Park H, Park HS, Choi EJ, Kim S. Glutamine-dependent antiapoptotic interaction of human glutaminyl-tRNA synthetase with apoptosis signal-regulating kinase 1. J Biol Chem 2001;276:6030-6

Kulpa J, Wo'jcik E, Reinfuss M, Kolodziejski L. Carcinoembryonic antigen squamous cell carcinoma antigen, CYFRA 21-1, and neuron-specific enolase in squamous cell lung cancer patients. Clin Chem 2002;48:1931-7

Kwon MS, Shin SH, Yim SH, Lee KY, Kang HM, Kim TM, Chung YJ. CD63 as a biomarker for predicting the clinical outcomes in adenocarcinoma of lung. Lung Cancer 2007; 57:46-53

Labouesse M. The yeast mitochondrial leucyl-tRNA synthetase is a splicing factor for the excision of several group I introns. Mol Gen Genet 1990;224:209-21

Lee EJ, Choi EM, Kim SR, Park JH, Kim H, Ha KS, Kim YM, Kim SS, Choe M, Kim JI, Han JA. Cyclooxygenase-2 promotes cell proliferation, migration and invasion in U2OS human osteosarcoma cells. Exp Mol Med 2007;39:469-76

Lee SW, Cho BH, Park SG, Kim SH. Aminoacyl-tRNA synthetase complexes: beyond translation. J Cell Science 2004;117:3725-34

Livak KJ, Schmittgen TD. Analysis of relative gene expression data using real-time quantitative PCR and the $2(-\Delta \Delta \mathrm{C}(\mathrm{T}))$ method. Methods $2001 ; 25: 402-8$

Müller-Hagen G, Beinert T, Sommer A. Aspects of lung cancer gene expression profiling. Curr Opin Drug Discov Devel 2004;7:290-303

Nguyen DM, Schrump DS. Lung cancer staging in the genomics era. Thorac Surg Clin 2006;16:329-37

Okada M, Nishio W, Sadamoto T, Uchino K, Yuki T, Nakagawa A, Tsubota N. Prognostic significance of perioperative serum carcinoembronic antigen in nonsmall cell lung cancer: analysis of 1,000 consecutive resections for clinical stage I disease. Ann Thorac Surg 2004;78:216-21

Otani A, Slike BM, Dorrell MI, Hood J, Kinder K, Ewalt KL, Cheresh D, Schimmel P, Friedlander M. A fragment of human TrpRS as a potent antagonist of ocular angiogenesis. Proc Natl Acad Sci USA 2002;99:178-83

Rho SB, Lincecum TL Jr, Martinis SA. An inserted region of leucyl-tRNA synthetase plays a critical role in group I intron splicing. EMBO J 2002;21:6874-81

Schneider J. Tumor markers in detection of lung cancer. Adv Clin Chem 2006;42:1-41 
Siemes C, Visser LE, Coebergh JW, Splinter TA, Witteman JC, Uitterlinden AG, Hofman A, Pols HA, Stricker BH. $\mathrm{C}$-reactive protein levels, variations in the $\mathrm{C}$-reactive protein G, and cancer risk: the Rotterdam Study. J Clin Oncol 2006;24:5216-22

Testa JR, Liu Z, Feder M, Bell DW, Balsara B, Cheng JQ, Taguchi T. Advances in the analysis of chromosome alterations in human lung carcinomas. Cancer Genet Cytogenet 1997;95:20-32
Wakasugi K, Slike BM, Hood J, Ewalt KL, Cheresh DA, Schimmel $P$. Induction of angiogenesis by a fragment of human tyrosyl-tRNA synthetase. J Biol Chem 2002;277: 20124-6

Zhang J, Ren H, Yuan P, Lang W, Zhang L, Mao L. Downregulation of hepatoma-derived growth factor inhibits anchorage-independent growth and invasion of non-small cell lung cancer cells. Cancer Res 2006;66:18-26 